\title{
Article
}

\section{On the optimization of maintenance storage cost in industry a fuzzy logic application}

Khalil, Jean and Labib, Ashraf W.

Available at http://clok.uclan.ac.uk/38112/

Khalil, Jean ORCID: 0000-0002-5476-0799 and Labib, Ashraf W. (2021) On the optimization of maintenance storage cost in industry a fuzzy logic application. International Journal of Quality \& Reliability Management . ISSN 0265-671X

It is advisable to refer to the publisher's version if you intend to cite from the work. http://dx.doi.org/10.1108/JJQRM-01-2021-0009

For more information about UCLan's research in this area go to http://www.uclan.ac.uk/researchgroups/ and search for < name of research Group>.

For information about Research generally at UCLan please go to http://www.uclan.ac.uk/research/

All outputs in CLoK are protected by Intellectual Property Rights law, including Copyright law. Copyright, IPR and Moral Rights for the works on this site are retained by the individual authors and/or other copyright owners. Terms and conditions for use of this material are defined in the policies page.

\section{CLoK}

Central Lancashire online Knowledge www.clok.uclan.ac.uk

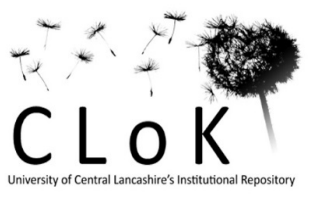




\title{
On the Optimization of Maintenance Storage Cost in Industry a Fuzzy-Logic Application
}

\author{
Jean Khalil (Corresponding Author)
}

ORCID ID 0000-0002-5476-0799

Department of Mechanical Engineering

\author{
Arab Academy for Science, Technology and Maritime Transport \\ Sheraton Complex, from El Mosheer Ahmed Ismail, Cairo, Egypt \\ Jean.khalil@aast.edu \\ Jmbkhalil@yahoo.co.uk \\ Mob:00201005174111 \\ Ashraf Labib \\ Operations and Systems Management Group, \\ University of Portsmouth, \\ Portsmouth, PO1 3DE \\ United Kingdom \\ $\underline{\text { Ashraf.labib@port.ac.uk }}$
}

\begin{abstract}
Purpose: The purpose of this paper is to construct a fuzzy logic model that acts as a decision support system to minimize inventory-related costs in the field of industrial maintenance. Achieving a balance between the unavailability and over-storage of spare parts is a problem with potentially significant consequences. That significance increases proportionally with the everincreasing challenge of reducing overall cost. Either scenario can result in substantial financial losses because of the interruption of production or the costs of tied-up capital, also called the "solidification of capital." Moreover, there is that additional problem of the expiry of parts on the shelf.
\end{abstract}

Approach: The proposed approach relies on inputs from experts with consideration for incompleteness and inaccuracy. Two levels of decision are considered simultaneously. The first is whether a part should be stored or ordered when needed. The second involves comparing suppliers with their batch-size offers based on user-determined criteria. A mathematical model is developed in parallel for validation.

Findings: The results indicate that the fuzzy logic approach is accurate and satisfactory for this application and that it is advantageous because of its limited sensitivity to the inaccuracy and/or incompleteness of data. In addition, the approach is practical because it requires minimal effort from the user.

Originality: To the best of the authors knowledge the exploitation of Fuzzy Logic altogether with limited sensitivity experts' inputs were never combined for the solution of this particular problem however this approach's positive impact is expected to be highly significant in solving a chronic problem in industry.

Keywords: Fuzzy logic modeling; Decision Support Systems; Industrial Maintenance; Cost Optimization; maintenance engineering 


\section{Introduction}

For industrial organizations, achieving optimal maintenance costs is critical for maximizing profits (Bousdekis, Papageorgioua, Magoutas, Apostolou, \& Mentzas, 2018) and (Antomarioni, Bevilacque, Potena, \& Diamantini, 2019). The downtime of machinery is one of the greatest sources of financial loss because of lost opportunities and/or penalties, including outsourcing the supply or service to ensure the customer requirements are met. The cost of downtime is proportional to the scale of the application, except in the case of redundancy. With variations of product models among other factors, a policy of redundancy may be more expensive than an immediate repair so it may represent a greater financial loss. Sleptchenko and Van der Heijden (2016) reported that the average cost of one hour of downtime for a commercial aircraft was approximately USD 10,000, and for a specific machine in the electronics industry, the cost is estimated to be 10 times that amount. It is common in industry and in-service operations to incur very significant downtime costs (Chanchal, Maiti, Shafiee, \& Kumaraswamy, 2018). In train operations, for instance, a slight delay in arrival could cost the company thousands of dollars in returned tickets and/or supplying other means of commuting for passengers. An Alstom maintenance manager contracted by West Midlands Railways in the UK explained to conference attendees that a broken bread toaster in a first-class compartment could cost the company thousands of pounds sterling. This was because the failure to serve breakfast to first-class passengers would require the company to refund the values of the passengers' tickets. Therefore, a broken toaster is sent in a special van to the manufacturer for immediate repair overnight. It is common knowledge that a key reason, and typically the primary reason, for long downtimes is the unavailability of spare parts.

The availability of too many spare parts is the other side of this problem, and it includes two cost factors. The first is the solidification of capital defined as financial losses incurred because funds are tied up in the form of unnecessary stored parts, in other words, the lost opportunity of investing this money. The second cost factor is the possible expiry of parts on the shelf. In industry, invested capital goes into a profit-making cycle, which starts with raw material and ends with sold products. The length of the cycle depends on the nature of the industry and, in many industries, the fiscal year includes multiple cycles. From this perspective, the solidification of capital is a direct loss to the operations (Syntetos, Teunter, Babai, \& Transchel, 2016). Moreover, in a number of developing countries, for numerous reasons, interest rates are extremely high. In those situations, the solidification of capital becomes a significant threat to the overall interests of the industrial organization. Mostafa, Dumrak, \& Soltan (2015) reported the relating of lean maintenance to lean manufacturing and included the variable of spare part unavailability as a direct cause of maintenance waste. It is common practice that decision-makers, fearing blame from their managers or even a penalty, resort to over-storage, which can be difficult to monitor, as opposed to the unavailability of parts, which is immediately apparent.

Because of this problem, there is an ongoing need to attain a nearly correct balance between unavailability and over-storage of spare parts. In this study, this balance considers solely the financial interests of the organization. It does not include strategic 
applications where availability and reliability must be maintained at the highest possible levels, regardless of cost. The proposed models consider a generic case of random failures with perfect repairs subject to the availability of spare parts.

A novel systematic approach is suggested, aiming to support relevant personnel in constructing a fuzzy logic model that proves effective in supporting decisions regarding:

1- The selection of the best policy for commissioning spare parts - either "Order at breakdown" or "Keep in store."

2- In either case and under different supply offers, supporting the selection of a supplier, given the financial benefit to the user.

Building up the model's rules relies on expert reasoning while implementing the model relies on user inputs. So, the latter exploits the power of fuzzy logic in tolerating inaccuracies, uncertainties, and missing data. Diluting the effect of imperfect inputs is a prime advantage of this approach. Moreover, the model's outcome is tested against a mathematical model, and it is shown to be valid. To the best of the authors' knowledge, such an approach has not been attempted in the literature.

\section{Literature Review}

Extensive studies were conducted to identify research that addressed the problem of inventory optimization, particularly in terms of supplier selection, commissioning policies, and optimizing the size of orders (Qiwei, Boylan, Huijing, \& Labib, 2018). Numerous studies have reported a strong relationship between proper management of the availability of spare parts and the profitability of production. The overarching perception in these studies is that, the greater the investment in assets, the greater is the impact of production disruption (Lengu, Syntetos, \& Babai, 2014), (Atieh, et al., 2016) and (Cusano \& Napoletano, 2017).

\subsection{Scope of the Approach and Aspects for Consideration}

The criticality of spare parts - specifically the effect of their shortage on production —is a crucial parameter in the arena of spare parts commissioning strategies. Stoll, Kopf, \& Lanza (2015) and Khalil (2010), studied it with different levels of attention. Both studies considered a three-dimensional assessment tool, but while the former used linear programming, the latter implemented the analytical hierarchy process (AHP) developed by Saaty Thomas as a scoring technique (L \& Saaty, 1990). This technique is frequently used in multi-criteria decision making (Ishizaka \& Labib, 2011). Labib (2011) also investigated the power of the AHP by comparing its performance to fuzzy logic to solve the problem of selecting a spare parts supplier. In a similar application, Antosz \& Chandima Ratnayake (2016) and Teixeira, Lopes, \& Figueiredo (2017) performed case studies where they produced a scoring technique for the significance of spare parts based on relevant criteria. Moreover, Chaklar, Ishizaka, Labib, \& Saad (2016) developed a multi-criteria decision methodology, which they applied to the classification of spare parts for optimized inventory management. On a similar note, Lopez-Soto, Angel-Bello, Yacout, \& Alvarez (2017) trained an artificial neural network (ANN) to classify a set of inventory items using the Pareto ABC analysis. That ANN was then able to accommodate newcomers and re-perform the classification.

Wen, Han, Yang, \& Kang (2017), Costantino, Di Cravio, Patriarca, \& Petrella (2018), and Zu, Dekker, Willem, Renjie, \& Koning (2017) approached the problem of spare parts stochastic demands not only in terms of unpredictability but also with consideration for data unavailability and the uncertainty of human judgment. Their attempts to address these problems relied on generic algorithms. The issue of forecasting the demand for spare parts has garnered attention from many other researchers. Van der Auweraer, Boute, \& Syntetos (2019) performed a review on the subject and concluded that most of the available literature relies on extrapolation rather than on strong input parameters. Hasni, Aguir, \& Babai (2018) reviewed the efficacy of the bootstrapping approach for the same issue. Lengu, Syntetos, \& Babai (2014) used compound distribution empirically to model the intermittent nature of the demand for spare parts demand. Boylan \& Babai (2016) empirically compared two methods to forecast spare parts demand uncertainty and found that the overlapping temporal demand aggregation is favorable over the non-overlapping one. Prak D. Teunter, Babai, Syntetos, \& Boylan (2018) used the method of moment's principles for a compound Poisson distribution to improve the forecasting capability of spare parts demand. On the track of demand modeling. Syntetos \& Teunter (2014) worked on the correction of error within the forecasting of the lead time to improve the efficacy of safety stocks level whereas Prak, Ruud, \& Syntetos (2017) considered the variation of demand in order to ensure a proper level of safety stocks. Furthermore, Rekik, Christoph, \& Syntetos (2017) used managerial inputs for the fine-tuning of replenishment policies. It is worth mentioning that managerial inputs in this context are very different than the experts' inputs which are in the core of the fuzzy logic approach that is used in this paper. Prak, Teunter, \& Riezebos (2014) compared periodic and continuous review of spare part levels to the 
periodic and continuous ordering of new spare parts and concluded that the best combination is periodic review and continuous ordering. Zahedi-Hooseini, Scarf, \& Syntetos (2017) and Zahedi-Hosseini, Scarf, \& Syntetos, (2018) linked routine inspection maintenance to ordering and replenishing spare parts. Using a near-similar concept (Moharana, Sarmah, \& Rathore, 2019) used a data mining approach that relies on sequential patterning to predict the bill of required spare parts.

\subsection{Consideration of the Effect of Shelf Life and Solidification of Capital}

In the literature or in practice, hardly any attention has been given to the financial impact of the shelf life of a part or to the negative impact of the solidification of capital in over-stored products. However, there have been exceptions. Kara \& Dogan (2018) developed a reinforcement learning program to optimize the ordering of perishable products such as food, beverages, and medication within the specific field of spare parts inventory optimization. Refurbishing spare parts is an acceptable solution, although it meets with a great deal of disfavors in industry. If it is used, much work is required to prove its feasibility (Yoo \& Kim, 2016).

A non-orthodox approach that limits the harmful effects of the solidification of capital is to store renewed spare parts to keep the equipment running while a new part is commissioned. This approach is valid under certain conditions: 1) The part must be a component or system where restoration is possible; 2) the new part is expensive; 3) the restoration is significantly cheaper than the original price and it restores the part to a satisfactory condition; 4) the failure pattern is unpredictable but costly, and 5) the replacement time is short compared to the lead time. One of the authors witnessed the success of this approach in a food factory in Wales. However, success is always subject to those five constraints. Sleptchenko \& Van der Heijden (2016) compared the standby cost and the stock of low-grade spare parts to optimize cost under the constraint of system availability. They used approximation and mathematical modeling, and they found that the approximation was reasonably accurate. Erkoc \& Ertogral (2016) considered a special case of applications that were primarily applicable to aircraft, where subsystems and sub-subsystems are completely independent of the device and have defined service intervals before mandatory replacement with an overhauled substitute. They researchers recommended that the company optimizes the pre-overhauling with respect to the capacity of the overhauling facility and queuing management. Somarin, Chen, Asian, \& Wang (2017) also considered the case of repairable spare parts that could be stored in different locations. They proposed a semi-Markov chain that optimized the selection of the most suitable store for the commissioning of parts and for the reconditioning of failed parts.

\subsection{Consideration of Experts' Judgments as Useful Inputs}

Researchers also considered specific versions of the problem. Schuh, Schnider, Funke, \& Tracht (2015) integrated a hazard model with expected environmental conditions to model the optimum quantities of spare parts for offshore wind turbines. Moharana \& Sarmah (2016) defined the term, "associated spare items," for the underlying relationships that could exist between groups of spare parts. Therefore, they could model the stocking policy for each group as a unit, because the failure of one item in the group could interrupt the implementation of the rest.

Baecke, De Baets, \& Vanderheyden (2017) combined statistical solutions with expert judgment solutions, but they applied this combination to supply chain management in general. They regarded this combination as not only beneficial but they believed it should be mandatory for practical solutions.

\subsection{Interpretation of the Literature Review and Formulation of the Problem under Consideration}

The bulk of the available studies assumed that the user would implement complex methods to achieve optimality. That assumption rarely holds true in a typical industrial organization. In fact, there exists a huge gap between theoretical and applicable approaches within the field (Naji, El Oumami, Bouksour, \& Beidouri, 2019). In addition, a wide majority of reviewed research relied on the assumption that the user had complete data and clear inputs. Although this assumption can be realistic at times, it is typically incorrect. The fuzziness of the inputs is the rule and not the exception, and quite frequently the user does not have full insight into the mathematics of their problem and/or of the suggested solutions. Moreover, the financial effects of the shelf life of a part and solidified capital are hardly given enough attention in the literature. Nevertheless, they are believed to be essential input factors that ensure the desired outcome of overall cost optimality. The aspect of currency inflation being a very considerable input parameter in numerous countries also has not been addressed in the literature.

Therefore, the question is how to optimize the overall cost of managing spare parts availability with consideration of relevant cost causal factors and under constrained data availability and accuracy; given two main alternatives namely stacking spare parts 
in store or ordering them at the event of breakdown. Moreover, how to accommodate the different cost causal parameters into in order to compare different suppliers and/or offers.

To the best of the authors' knowledge, no study has approached the problem under consideration by developing and validating a simple inference decision support system that exploits the power of fuzzy logic and accommodates missing and/or inaccurate inputs. The work presented in this paper offers support to the role of the user. Thus, it maximizes the contribution of human expertise by taking advantage of fuzzy logic to solve this critical problem. In addition, A MATLAB code is built for the proposed fuzzy logic decision support system as well as a crisp mathematical model to validate it. Both models are implemented in parallel for purposes of comparison. Both approaches have been manually verified and studied in terms of sensitivity.

\section{Proposed Solution}

The problem under consideration may be stated this way: in the control of spare parts inventory, users must identify the cost impact of ordering a part from different suppliers at the event of a breakdown as opposed to stocking the part at different commissioning policies and/or from different suppliers, given the relevant cost factors in all cases. Factors like the shelf life of parts, cost of solidification of capital and suppliers' ability to abide by their promised lead times are typically omitted in the literature, but they are believed to be crucial in a wide range of applications. Any proposed solution must satisfy these objectives, and it must prove to be correct and valid.

Before formulating the proposed solution, the following assumptions are made:

1- The spare parts under consideration cater to perfect repair actions by part replacement.

2- Within the context of this study, the term "downtime" refers to the lead time, being the amount of time lost while waiting for the spare part; it does not include any other elements of machine downtime.

3- The economic and political conditions where the organization is located are embedded in the inputs of the user. For example, traffic in Europe is different from that in central Africa. This would affect the lead time and the "degree of compliance" to that lead time. Such eventualities are to be considered by the modeler in the input data.

4- The study must be performed within a defined time horizon because probabilities and numbers of occurrences, among other causal factors, must be constrained within a defined time period.

\subsection{Mathematical Solution}

In this section, a mathematical model is proposed to address the situations under consideration. Table I presents the variables that comprise the relationships of the proposed mathematical solution, where $\mathrm{k}$ and i refer to the suppliers of "Keep in store" and "Order at breakdown," respectively. More inputs like the consumption rate/hour are fed by the user through the model interface and are included in the calculations of the variables $\mathrm{X}, \mathrm{Y}$, and $\mathrm{Z}$. These calculations take place in parallel to feed the relevant variables of the mathematical equations (1 to 6).

The input "batch size" is valid only for the "Keep in store" alternatives, whereas for the "Order at breakdown" alternatives, the batch size is irrelevant and typically equals 1 . Given the relevant variables, mathematical relationships are developed.

The cost of keeping an item (k) in store comprises the cost of expiry and the cost of solidification.

- Additional Cost per Batch $(k)=$ Cost of Expiry $(k)+$ Cost of Solidification of capital $(k)$

- $\quad$ Cost of Expiry $(k)=Z \times$ Initial Price per Part $(k) \times(1+$ Interest Rate $)$

- $\quad$ Cost of Solidification of capital $(k)=$

$$
(\text { Batch Size }(k)-Z) \times \text { Initial Price per Part }(k) \times \frac{X}{2} \times \text { Interest Rate }
$$

\section{Equation 3}

Equation 1 "Additional Cost per Batch" presents the additional cost of keeping an item in store per full batch. Equation 2 presents the losses that are caused by expired parts on the shelf. Equation 3 presents the losses that are caused by the solidification of capital on the shelf, also called tied-up capital. For a given batch of spare parts, if the whole batch is fully depleted in the period of time $\mathrm{X}$ and if the rate of consumption is constant, then the average period of time on the shelf per piece is $\frac{X}{2}$. During 
this period, the cost of the parts is tied up (solidification of capital). The larger the batch size, the higher the solidification of capital.

Equation 4 presents the additional cost of keeping one item in store.

$$
\text { Additional Cost per Part }(k)=\frac{\text { Additional Cost per Batch }(k)}{(\text { Batch Size }(\boldsymbol{k})-Z)}
$$

\section{Equation 4}

Therefore, the total cost of keeping a single item part in store is:

Overall Cost per Part $(k)=$ Initial Price per Part $(k)+\operatorname{Additional~Cost~per~Part~}(k)$

Equation 5

The input factor "Interest Rate" is added to 1 because the original value of the part at the time of expiry will be lost. This is in addition to the cost of losing the opportunity to invest the initial amount in production.

Conversely, the cost of ordering the item in the event of a breakdown, given multiple suppliers, is as follows:

$$
\operatorname{Cost}(i)=\left[\frac{C p(i-M)}{T l(i-M)}+C m i\right] \times \operatorname{Tl}(i-M) \times \frac{P_{f}}{A_{s}(i-M)}
$$

Equation 6

where $\mathrm{i}$ and $\mathrm{M}$ form the loop that calculates the cost per supplier resulting in a cost function of (i), which is the identification number of the supplier.

The system does not make a decision. Rather, by performing the necessary calculations, it presents the results to the user, who

\begin{tabular}{|c|c|c|}
\hline Variable & Definition & Unit \\
\hline \multicolumn{3}{|c|}{ Input data for the "Keep in store" policy } \\
\hline $\begin{array}{l}\text { Initial price } \\
\text { per Part (k) }\end{array}$ & Initial price/part/supplier & monetary unit \\
\hline Batch size (k) & Number of commissioned parts per supplier & part \\
\hline $\begin{array}{l}\text { Consumption } \\
\text { rate }(k)\end{array}$ & Rate of consumption of the part per unit of time & part $/ \mathrm{h}$ \\
\hline Shelf life (k) & Time to the expiry of a part & $\mathrm{h}$ \\
\hline Interest rate & Capital interest per hour (Net profit -Inflation) & $\begin{array}{l}\text { monetary } \\
\text { unit/h }\end{array}$ \\
\hline \multicolumn{3}{|c|}{ Input data for the "Order at breakdown" policy } \\
\hline Cp(i) & $\begin{array}{l}\text { With respect to the cheapest offer, the increase in price from another vendor who promises } \\
\text { less lead time and/or who has a higher probability to abide by their claimed lead time. }\end{array}$ & monetary unit \\
\hline Tl(i) & Lead time per supplier & $\mathrm{h}$ \\
\hline Cmi & Cost of machine idleness per hour & $\begin{array}{l}\text { monetary } \\
\text { unit/h }\end{array}$ \\
\hline$P_{f}$ & Probability that item will fail within the constrained domain of study. & \\
\hline$A_{s}$ & $\begin{array}{l}\text { Degree to which supplier abides by their theoretical lead time. Calculated as probability } \\
\text { (Degree of Compliance) }\end{array}$ & \\
\hline
\end{tabular}
will decide accordingly. 


\begin{tabular}{|l|l|c|}
\hline \multicolumn{2}{|c|}{ Calculated variables by the programed model “Keep in store” policy } & \\
\hline T-shelf $(\mathbf{k})$ & Amount of time a part remains on the shelf until used & $\mathrm{h}$ \\
\hline $\mathbf{X}(\mathbf{k})$ & Time until the whole batch is used & $\mathrm{h}$ \\
\hline $\mathbf{Z}(\mathbf{k})$ & Number of parts that will expire (out of given commissioned batch size) & part \\
\hline
\end{tabular}

\subsection{The Fuzzy Logic Approach}

Mathematical solutions have drawbacks, the most critical of which is that they require users to have a thorough understanding of the problem as well as accurate input data. Typically, in industry, users prefer systems that remain reasonably valid with limited data and possibly compromised accuracy of inputs. For this reason, the fuzzy logic method first developed by Lotfy Zada (Zada, 1965) has been widely implemented in a diversity of fields (Komal, 2019), (Samima, Sarma, Samanta, \& Prasad, 2019), (Zare, Malakoutikhah, \& Alimohammadou, 2019) and (Jatoba, Cesar Bellas, Koster, Burns, \& Vidal, 2018). Fuzzy logic was also implemented heavily in the field of industrial maintenance and reliability (Rashid, Place, \& Braithwaite, 2014), (Sene, Kamsu-Foguem, \& Rumeau, 2018) and (Tillement \& Hayes, 2019). Fuzzy logic enhances the decision-making capacities of humans by transforming their knowledge and expertise into satisfactory recommendations with respect to their intentions. In this study, the proposed fuzzy logic model involves three cost components, which are inputted by users and experts and are specific to the particular failure mode:

1- $C_{f}$ : According to the financial impact on production that is caused by the unavailability of the part, the added cost of lead time per supplier divided by the degree of confidence that this supplier can comply with their claimed lead time. This latter parameter is a probability digit that ranges between 0 and 1 , where 1 is $100 \%$ compliance.

2- $C_{s}$ : According to the financial impact on the industrial organization, the added cost that accounts for the solidification of capital when storing a batch of parts for a given period of time.

3- $C_{e}$ : According to the financial impact on the industrial organization, the added cost of storing the part in terms of expiry on the shelf. This value is entered for each supplier given the batch size they offer. If the same supplier has two offers, then they are entered as two entries, each with its specific values.

Once these values are entered, the code reads, calculates, and stores two arbitrary values for normalization.

1- $C_{\text {part-min }}$ : The minimum value of the three parameters for all suppliers.

2- $C_{\text {part-max }}$ : The maximum value of the three parameters for all suppliers.

The calculated $C_{\text {part-min }}$ and $C_{\text {part-max }}$ constitute the lower and upper limits of cost, against which every cost component for each supplier is normalized. This results in values that are between 0 and 1 both included. The normalized added cost components $x_{1}, x_{2}$, and $x_{3}$ are then calculated by Equations 7, 8, and 9 respectively, and they make up the normalization vectors of the added cost components through the fuzzy system.

- $x_{1}=\frac{C_{f-} C_{\text {part }-\min }}{C_{\text {part }-\max }-C_{\text {part }-\min }}$

- $x_{2}=\frac{C_{s}-C_{\text {part }-\min }}{C_{\text {part }-\max -}-C_{\text {part }-\min }}$

- $x_{3}=\frac{C_{e-} C_{p a r t-\min }}{C_{p a r t-\max }-C_{\text {part }-\min }}$
Equation 7

Equation 8

Equation 9

The three values are then arranged in a single vector

- $x=\left[\begin{array}{lll}x_{1} & x_{2} & x_{3}\end{array}\right]$

Equation 10 
The normalized input values are fuzzified through memberships that consist of triangular, homogeneous, and equidistant fuzzy sets from 0.1 to 0.9 (see Figure I).

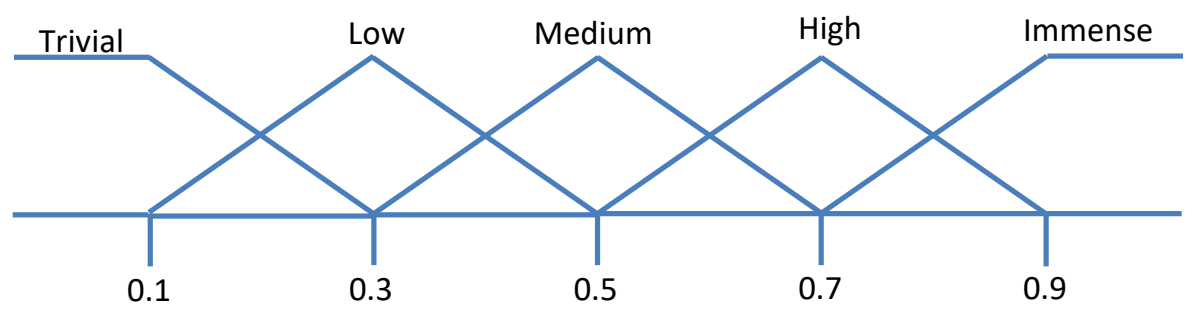

Figure 1: Fuzzification membership function.

Table 1. Input variables for the mathematical approach to the case study

\begin{tabular}{|c|c|c|}
\hline Variable & Value & Unit \\
\hline \multicolumn{3}{|c|}{ "Keep in Store" } \\
\hline Initial Price per Part & {$[569.2 ; 690 ; 790]$} & monetary unit \\
\hline Batch Size & {$[48 ; 36 ; 12]$} & part \\
\hline Consumption Rate & 0.002083 & part/hour \\
\hline Shelf Life & 15120 & hour \\
\hline Interest Rate & 0.000547945 Monetary & unit/hour \\
\hline \multicolumn{3}{|c|}{ "Order at Breakdown" } \\
\hline Actual price of part & {$[2510 ; 1280 ; 810]$} & monetary unit \\
\hline Added cost of delivery $C p(i)=$ & $\begin{array}{c}{[2510-569.2 ; 1280-569.2 ; 810-569.2]} \\
{[1940.5 ; 710.8 ; 240.8]}\end{array}$ & monetary unit \\
\hline Tl(i) & {$[10 ; 24 ; 48]$} & hour \\
\hline Cmi & 400 & monetary unit/ hour \\
\hline Pf & 0.25 & \\
\hline Degree of Compliance & {$[0.75 ; 0.95 ; 0.85]$} & \\
\hline
\end{tabular}

The fuzzified values are processed within the rule base tables (Appendix 1), which are arranged as follows:

Five tables: each table presents one interval of the variable "Cost of failure."

Five rows in every table: each row presents one interval of the variable "Cost of expiry."

Five columns in every table: each column presents one interval of the variable "Cost of solidification."

In fuzzy logic, the area of the rule base table is a fiddly part of modeling. Their main function is to reflect the user's views and preferences. Therefore, if built abstractedly, they may misrepresent the results. The following steps show a suggested scorebased assistance technique to build the rule base tables. 
1- The fuzzy sets are given respective weights of 1 to 5 , trivial being equal to 1 and immense equal to 5 . This weight is merely used for building the table cells. It should not be confused with the actual numerical values of the fuzzy sets which are used in memberships' computation.

2- The weights of the two factors "Cost of expiry and "Cost of solidification" are summed in a score that represents the impact of the "Keep in store" policy.

3- In every cell, the ratio between the weight-scores of "Order at breakdown" and "Keep in store" is annotated.

4- The ratios are normalized as per the lower weight (e.g. 2:7 is 1:3.5).

5- Score ratios are tabulated in separate tables (Appendix II.1)

6- A set of outcome fuzzy sets (OFS) is suggested. To prevent the same OFS in two consecutive cells in either direction, the number of OFS is set to accommodate the number of different ratios (Appendix II.2). The main domain is between 0.05 and 0.95, but extreme values are assigned numerical outcome values as in the table shown in Appendix II.

7- The obtained output truth values are de-fuzzified on a homogeneous equidistant membership function where five fuzzy set outputs are distributed evenly between 1 and 9 , while each fuzzy set is further divided into three values namely, Lower (L), Middle (unlabeled) and Upper (U) as in the figure A. II (Appendix II)

With the accommodation of the extreme values, the resulting output is a number between 0 and 1 . The lower the score the more favorable this particular "Order at breakdown" with respect to this particular "Keep in store." The model is designed to run every alternative of one policy (Order at breakdown) to all alternatives of the other policy (Keep in store). So, a table of output values is created, showing the most favorable and the second most favorable alternatives.

\section{Case Study}

This section demonstrates the implementation of both approaches on an actual item from a satellite plant of a giant multinational automotive company where domestic vehicles are assembled. The item is an imported chemical that is applied to treat machine parts before special lubricants are applied. The real data are complemented by some assumptions to produce a comprehensive and explanatory case study that draws a full picture of the approaches. The shelf life of this material is 21 months. The consumption rate is around 12 pieces per month. In the case of "Keep in store," the part may be ordered in different batch sizes with respective prices per unit. In the case of "Order at breakdown," the part may be ordered through different shipment policies at different lead times, degrees of compliance, and costs. It is estimated that the non-availability of the item costs 400 monetary unit/h in lost opportunity of production.

\subsection{The Mathematical Approach}

Table II shows the inputs of the case in terms of the variables for the mathematical approach.

The mathematical model computed the inputs and yielded the data in Table III and Figure II. Each alternative is represented by a cost bar on the chart. Furthermore, for each alternative of "Order at breakdown," there are two bars that represent the lead time of the supplier and their degree of compliance.

Table 2. Results of the mathematical approach to the case study

\begin{tabular}{|c|r|r|r|r|r|r|}
\hline & \multicolumn{1}{|c|}{ Store 1 } & Store 2 & \multicolumn{1}{c|}{ Store 3 } & Alternative 1 & Alternative 2 & Alternative 3 \\
\hline Cost & $3.2255 \mathrm{e}+03$ & $3.6471 \mathrm{e}+03$ & $2.0369 \mathrm{e}+03$ & $1.9802 \mathrm{e}+03$ & $2.7134 \mathrm{e}+03$ & $5.7179 \mathrm{e}+03$ \\
\hline Lead Time & 0 & 0 & 0 & 10 & 24 & 48 \\
\hline Confidenace Rate & 0 & 0 & 0 & 0.7500 & 0.9500 & 0.8500 \\
\hline
\end{tabular}




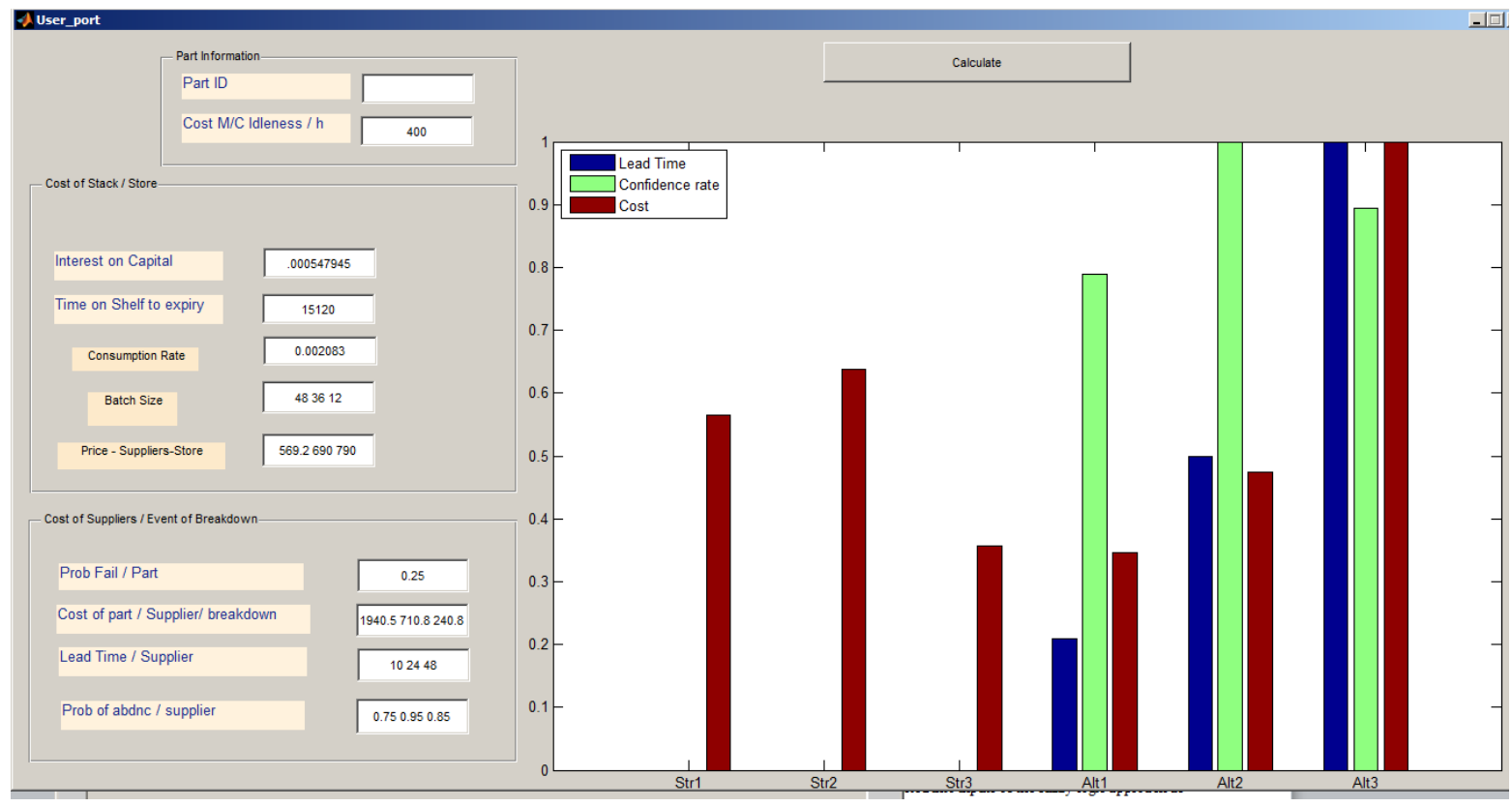

Figure 2. Results of the mathematical approach to the case study.

\subsection{The Fuzzy Logic Approach}

To compare the performance of the fuzzy logic approach and the mathematical approach, the inputs of the mathematical solution were converted into inputs for the fuzzy logic solution (Table IV). This accurately calculated conversion is conducted only for testing and validation purposes. In practice, fuzzy logic is typically required when accurate figures are not available. The run of the fuzzy model yielded the values in Table V.

Table 3. Input variables for the mathematical approach converted into the input format of the fuzzy logic approach

\begin{tabular}{|c|c|c|}
\hline Variable & Value & Unit \\
\hline \multicolumn{3}{|c|}{ "Keep in Store" } \\
\hline Cost of the part alone & {$[569.2 ; 690 ; 790]$} & monetary unit \\
\hline Batch size & {$[48 ; 36 ; 12]$} & part \\
\hline Number of expired parts & {$[16.5 ; 4.5 ; 0]$} & \\
\hline Number of non-expired parts & {$[31.5 ; 31.5 ; 12]$} & \\
\hline Saving per part & {$[220.8 ; 100 ; 0]$} & monetary unit \\
\hline Added cost of expiry & {$[9399.8 ; 3110 ; 0]$} & monetary unit \\
\hline $\begin{array}{l}\text { Added cost of expiry/part (divided by the } \\
\text { number of non-expired parts only) }\end{array}$ & {$[296.5 ; 98.7 ; 0]$} & monetary unit/part \\
\hline Added cost of solidification/non expired part & {$[74262 ; 90022 ; 14963]$} & monetary unit \\
\hline Added cost of solidification/part & {$[2357.5 ; 2857.8 ; 1246.9]$} & monetary unit/part \\
\hline $\begin{array}{c}\text { Actual cost of solidification/part } \\
\text { (Cost of solidification - saving)/part }\end{array}$ & {$[2136.7 ; 2757.8 ; 1246.9]$} & monetary unit/part \\
\hline \multicolumn{3}{|c|}{ "Order at Breakdown" } \\
\hline Cost of machine idleness & 400 & monetary unit/hour \\
\hline Probability of occurrence & 0.25 & \\
\hline Original price of the part & 569.2 & monetary unit \\
\hline Lead time & {$[10 ; 24 ; 48]$} & hour \\
\hline Cost of loss of production during lead time & {$[4000 ; 9600 ; 19200]$} & monetary unit \\
\hline Added cost of delivery & {$[2510-569.2 ; 1280-569.2 ; 810-569.2]$} & monetary unit \\
\hline
\end{tabular}




\begin{tabular}{|l|c|c|}
\hline \multicolumn{1}{|c|}{ Variable } & Value & Unit \\
\hline $\begin{array}{l}\text { Cost of loss of production + added cost of } \\
\text { delivery }\end{array}$ & {$[1940.5 ; 710.8 ; 240.8]$} & monetary unit \\
\hline
\end{tabular}

Table 4. Output truth values of the fuzzy logic approach

\begin{tabular}{|c|c|c|c|}
\hline & II-1st “Keep in store” & III-2nd “Keep in store” & I 3rd "Keep in store” \\
\hline I 1st “Order at breakdown" & 0.3187 & 0.2078 & 0.4563 \\
\hline II 2nd “Order at breakdown" & 0.4147 & 0.3712 & 0.5375 \\
\hline III 3rd “Order at breakdown" & 0.6131 & 0.5588 & 0.6705 \\
\hline
\end{tabular}

\subsection{Interpretation of the Output Table}

Since every alternative of "Keep in store" is compared to the same three "Order at breakdown" alternatives, the whole results matrix must be read simultaneously. When comparing the three "Keep in store" columns, it is obvious that the third column is the best alternative and is therefore marked with Roman numeral I. By comparing the three "Order at breakdown" rows, it is obvious that the first row is the best alternative. The differential comparison is therefore between the third "Keep in store" and the first "Order at breakdown." This equals an output truth value of 0.4563 , evidently less than the neutrality value 0.5 . This means that the best option is the first "Order at breakdown."

If it was required to identify the second-best option, the first "Order at breakdown" is removed from the table and the same procedure is followed. It indicates that the $3^{\text {rd }}$ "Keep in store" is the second-best option, since comparing it to the $2^{\text {nd }}$ best "Order at breakdown" (marked with II), the output is greater than 0.5 . The result also shows that the difference in favorability between the best and the second-best options is not large, as the absolute deviations from 0.5 are 0.0437 and 0.0375 , respectively. 


\section{Testing and Sensitivity Analysis}

Both models were extensively tested with scenarios that cover possible limits of the application. Only a few of these tests are included in this paper. In terms of verification, every run presented was confirmed by manual calculations. With regard to sensitivity, the mathematical approach consisted of first-degree relations, so input and output variations were strictly proportionate. The fuzzy logic is very different in that sense. As its name suggests, the effect of input variation is diluted by its process, and it is this characteristic that gives it an advantage in processing inaccurate data.

\subsection{The Sensitivity of the Fuzzy Logic Approach}

In consideration of the case study, input errors were introduced to the three "Order at breakdown" alternatives with the following deviation values $(0.05,0.1,0.15,0.20$, and 0.25$)$. The values of "Keep in Store" were kept unchanged. The resulting outputs with their attributed statistical identities are shown in Table VI and Table VII. Table VI shows the original output values in the second column and the output with an input deviation-error of 0.05 in the third column. The differences between those two values are in the fourth column, and so forth. Table VII extracts the differences and shows a statistical analysis of their values.

Table 6. Output values for the case study with assigned input errors

\begin{tabular}{|c|c|c|c|c|c|c|c|c|c|c|c|}
\hline & Original & $+5 \%$ & delta5 & $+10 \%$ & delta10 & $+15 \%$ & delta15 & $+20 \%$ & delta20 & $+25 \%$ & delta25 \\
\hline & \multicolumn{10}{|c|}{ 1st Keep in Store } & \\
\hline 1st Order at Breakdown & 0.1899 & 0.2147 & 0.0248 & 0.237 & 0.0473 & 0.258 & 0.0679 & 0.277 & 0.0868 & 0.294 & 0.1041 \\
\hline 2nd Order at Breakdown & 0.4459 & 0.4549 & 0.009 & 0.463 & 0.0174 & 0.471 & 0.0249 & 0.478 & 0.0318 & 0.484 & 0.0382 \\
\hline \multirow[t]{2}{*}{ 3rd Order at Breakdown } & 0.5802 & 0.5906 & 0.0104 & 0.6 & 0.02 & 0.609 & 0.0286 & 0.617 & 0.0366 & 0.624 & 0.0439 \\
\hline & \multicolumn{10}{|c|}{ 2nd Keep in Store } & \\
\hline 1st Order at Breakdown & 0.0943 & 0.1013 & 0.007 & 0.108 & 0.0134 & 0.126 & 0.0319 & 0.151 & 0.0562 & 0.172 & 0.078 \\
\hline \multirow[t]{2}{*}{ 3rd Order at Breakdown } & 0.5331 & 0.5399 & 0.0068 & 0.546 & 0.0129 & 0.553 & 0.0202 & 0.564 & 0.0305 & 0.573 & 0.0399 \\
\hline & \multicolumn{10}{|c|}{ 3rd Keep in Store } & \\
\hline 1st Order at Breakdown & 0.3898 & 0.3987 & 0.0089 & 0.407 & 0.017 & 0.414 & 0.0244 & 0.421 & 0.0312 & 0.427 & 0.0374 \\
\hline 2nd Order at Breakdown & 0.5773 & 0.5841 & 0.0068 & 0.591 & 0.0134 & 0.597 & 0.0192 & 0.602 & 0.0246 & 0.607 & 0.0295 \\
\hline 3rd Order at Breakdown & 0.6609 & 0.6639 & 0.003 & 0.667 & 0.0058 & 0.669 & 0.0083 & 0.672 & 0.0106 & 0.674 & 0.0128 \\
\hline
\end{tabular}

Table 7. Statistical identities of the output values with the assigned input deviation errors

\begin{tabular}{|c|c|c|c|c|c|c|}
\hline & delta5 & delta10 & delta15 & delta20 & delta25 \\
\hline & \multicolumn{5}{|c|}{ 1 $^{\text {st }}$ “Keep in Store” } \\
\hline 1st Order at Breakdown & 0.0248 & 0.0473 & 0.0679 & 0.0868 & 0.1041 \\
\hline 2nd Order at Breakdown & 0.009 & 0.0174 & 0.025 & 0.0318 & 0.0382 \\
\hline 3rd Order at Breakdown & 0.0104 & 0.02 & 0.029 & 0.0366 & 0.0439 \\
\hline & \multicolumn{7}{|c|}{$2^{\text {nd }}$ “Keep in Store" } \\
\hline 1st Order at Breakdown & 0.007 & 0.0134 & 0.0319 & 0.0562 & 0.07798 \\
\hline 2nd Order at Breakdown & 0.0154 & 0.0276 & 0.0408 & 0.0526 & 0.0611 \\
\hline 3rd Order at Breakdown & 0.0068 & 0.0129 & 0.0202 & 0.0305 & 0.0399 \\
\hline \multicolumn{7}{|c|}{$3^{\text {rd }}$ “Keep in Store" } \\
\hline 1st Order at Breakdown & 0.0089 & 0.017 & 0.0244 & 0.0312 & 0.0374 \\
\hline 2nd Order at Breakdown & 0.0068 & 0.0134 & 0.0192 & 0.0246 & 0.0295 \\
\hline 3rd Order at Breakdown & 0.003 & 0.0058 & 0.0083 & 0.0106 & 0.0128 \\
\hline \multicolumn{7}{|c|}{ Statistical parameters } \\
\hline Min & 0.003 & 0.006 & 0.008 & 0.011 & 0.013 \\
\hline Max & 0.025 & 0.047 & 0.068 & 0.087 & 0.104 \\
\hline Mean & 0.010 & 0.019 & 0.030 & 0.040 & 0.049 \\
\hline SD & 0.006 & 0.011 & 0.016 & 0.021 & 0.026 \\
\hline
\end{tabular}


In this context, the percentage of the deviation is not only irrelevant but misleading, because it relates to the original value quantitatively while it is a directional bipolar allocation vector. For example, a 0.01 deviation from 0.2 has the same effect as a 0.01 deviation from 0.8 as illustrated in Figure III.

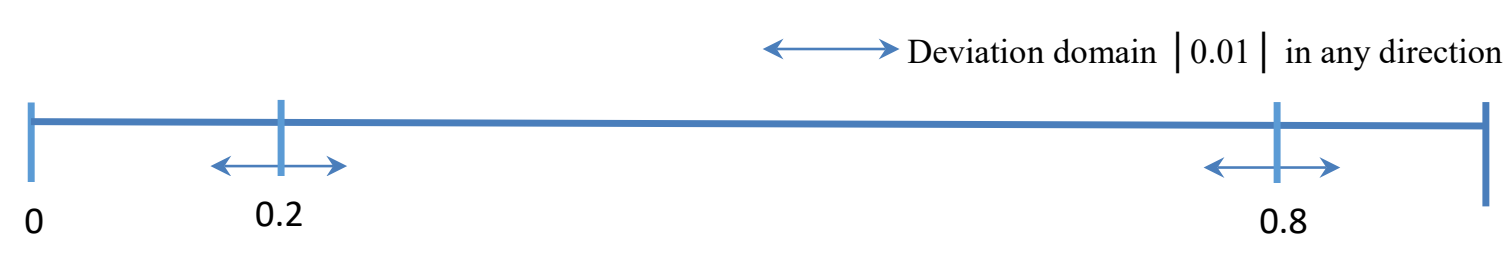

Order at Breakdown

Keep in store

Figure 3. Illustration of the effect of output deviation regardless of the original value

The most important finding of the sensitivity test is that the relativity of the outputs to each other remains intact. Therefore, the final arrangement of the favorability of the alternatives will not be affected by the input error. In other words, the user will always have a correct indication of the right decision. Many similar sensitivity tests were conducted, and those results showed that the outcome of the model remained consistent. Those tests also showed that the model outputs were dependent on the input within an acceptable margin of error. This is precisely the advantage of fuzzy logic in applications where exact figures are not easy to obtain.

As noted earlier, no model was found in the literature that targeted the same aims as the proposed model. Thus, it was not possible to test the performance of the proposed model against similar ones. However, since the mathematical model was verified by manual calculations, it follows that the fuzzy logic model was validated with respect to the crisp outputs of the mathematical model.

Thus, it can be concluded that, the fuzzy logic approach is able to damp input errors and variations because it takes inputs as abstract membership functions. As a consequence, it delivers results that correctly address the problem of developing a parts supply policy, selecting suppliers, and determining the size of an order. The approach is therefore validated as a decision support tool for the problem under consideration.

\subsection{One Step Further}

Within the fuzzy logic approach, the user can enter a relative weight between the two storage cost input-variables, namely "Cost of expiry" and "Cost of solidification." For example, a user may decide that the cost of expiry is twice as important as the cost of solidification because expired parts are a total waste but solidified capital is not as important to them. For this capability, the model interface includes a slide ruler that converts relative weights into proportions. To test the effect of this potential variation in inputs, a sub-code was written to run with relative weights from $1: 1$ to $3: 1$, in increments of 0.1 . The results of these tests confirmed the validity of the approach.

\subsection{The Ergonomics of the Solution}

Experience says that users in industry seldom choose complicated solutions. The friendliness of the proposed solution is believed to be an important point of strength. The MATLAB interface is designed to be concise and clear. Moreover, it requires the user to input a minimal amount of data and it has a tolerance for inaccuracies. 


\section{Limitations}

The fuzzy logic approach relies on the relationships between costs that were attributed to different policies about replacement parts and other factors like suppliers' ability to abide by their lead times. Because of this, the approach was not designed to compare one alternative to another. In that eventuality, the decision would be clear and would not require a decision support system. Also, the model is useful only when the differences - together with the causal factors - are close enough to cause ambiguity to the decision taker. If the values are well apart and the conclusions are obvious, then the model will not offer valuable help as the output values would tend to the two extreme values, 0.05 and 0.95 .

The fuzzy approach is not intended to be used with highly expensive parts such that a small error could result in significant financial losses. In such cases, the mathematical approach would be the correct choice because the cost of using it would be recovered by the accuracy of the calculation.

\section{Discussion}

There is a gap between solutions in the literature and in actual solutions. This is typically caused by the overall complexity of theoretically developed solutions and the preference of industrial personnel to resort to simpler solutions, particularly those that require only abstract inputs rather than calculated exact values. For a solution to be widely applicable, it is normally required to be practical. The survey of typical industrial sites showed that many of them still require a practical, yet usable, tool to solve the type of problem under consideration. It is believed that the proposed solution satisfies this requirement.

The user inputs, such as the actual cost of failure, are typically rough estimates because an accurate calculation is both costly and time-consuming. Mathematical formulations emphasize the inaccuracy of these estimations in their results, whereas fuzzy logic damps them, which, more frequently than not, yields better results. Expensive studies may be justified only in large applications where the investment in accurate sophisticated solutions will be recouped.

The work presented in this study considered the local nature of the application, using inputs from the actual experience of experts. This aspect adds a component of validity to the work.

This study showed that the complexity, the ambiguity, and the cost of a solution may be replaced by a simpler, clearer, and cheaper-yet equally efficient_-approach given the contribution of the user. In such a context, the assistance provided by a fuzzy logic model praises the power of human cognition and exploits its capacity.

The rule-based tables from which the fuzzy logic retrieved judgment values were hypothesized by the authors, and they may be tailored for specific uses. Even within these hypotheses, the user is given control over the relative weight of the two factors that contribute to the cost of storage.

\section{Conclusion}

In this study, a decision support tool that consists of two parallel approaches, one mathematical and one based on fuzzy logic, was proposed for the cost-optimization of the crucial subject: a supply strategy for spare parts within the industrial maintenance domain. Each approach acted as a decision support tool for the differential questions: 1) whether to order the spare part when needed or to keep it in stores, and 2) in any case, which supply offer was cost-optimal given relevant variables that were omitted in previous studies. With multi-causal factors contributing to the complexity of each question, each one was solved mathematically and through a fuzzy logic approach. The paper offered a systematic procedure for building the fuzzy logic model to enhance its validity. The results showed that the easier-to-apply fuzzy logic approach not only yielded results that were characteristically acceptable compared to the crisp mathematical approach, but it also permitted less extensive and less accurate data inputs. The fuzzy logic approach was also advantageous in terms of functioning with abstract inputs while damping input errors and approximations. In contrast to this, the accuracy of the mathematical approach was a function of the accuracy of its inputs. For the problem under consideration, complete and accurate input data are seldom available. The sensitivity and validity of both approaches were investigated and discussed. This work substituted complications of conventional solutions with the power of human cognition. It is expected that the outcome of this work would contribute significantly towards a universal optimized-solution for the problem under consideration according to the local conditions of the application. 


\section{References}

Antomarioni, S., Bevilacque, M., Potena, D., \& Diamantini, C. (2019). Defining a data-driven maintenance policy: an application to an oil refinery plant. International Journal of Quality \& Reliability Management, 36(1), 77-97.

Antosz, K., \& Chandima Ratnayake, R. M. (2016). Classification of spare parts as the element of a proper realization of the machine maintenance process and logistics - Case study. International Federation of Automatic Control, 49(12), pp. 1389-1393.

Atieh, A. M., Kaylani, H., Al-abdallat, Y., Qaderi, A., Ghoul, L., Jaradat, L., \& Hdairis, I. (2016). Performance Improvement of Inventory Management System Processes by an Automated Warehouse Management System. Computers in Industry, 41, 568-572.

Baecke, P., De Baets, S., \& Vanderheyden, K. (2017). Investigating the added value of integrating human judgment into stastical demand forecasting systems. International Journal of Production Economics, 191, 85-96.

Bousdekis, A., Papageorgioua, N., Magoutas, B., Apostolou, D., \& Mentzas, G. (2018). Enabling condition-based maintenance decisions with proactive event-driven computing. Computers in Industry, 100, 173-183.

Boylan, J. E., \& Babai, M. Z. (2016). On the performance of overlapping and non-overlapping temporal demand aggregation approaches. International Journal of Production Economics, 181, 136-144.

Chaklar, S., Ishizaka, A., Labib, A., \& Saad, I. (2016). Dominance-based rough set approach for group decisions. European Journal of Operational Research, 251, 206-224.

Chanchal, G., Maiti, J., Shafiee, M., \& Kumaraswamy, K. (2018). Reduction of life cycle costs for a contemporary helicopterthrough improvement of reliabiity and maintainability parameters. International Journal of Quality \& Reliability Management, 35(2), 545-567.

Costantino, F., Di Cravio, G., Patriarca, R., \& Petrella, L. (2018). Spare parts management for irregular demand items. Omega, 81, 57-66.

Cusano, C., \& Napoletano, P. (2017). Visual recognition of aircraft mechanical parts for smart maintenance. Computers in Industry, 86, 26-33.

Erkoc, M., \& Ertogral, K. (2016). Overhaul planning and exchange scheduling for maintenance services with rotable inventory and limited processing capacity. Computers and Industrial Engineering, 98, 30-39.

Hasni, M., Aguir, M. S., \& Babai, M. Z. (2018). Spare parts demand forecasting: a review onbootstrapping methods. International Journal of Production Research. doi:10.1080/00207543.2018.1424375

Ishizaka, A., \& Labib, A. (2011). Selection of new production facilities with the Group Analytic Hierarchy Process Ordering method. Expert Systems with Applications, 38, 7317-7325.

Jatoba, A., Cesar Bellas, H., Koster, I., Burns, C. M., \& Vidal, M. C. (2018). Supporting decision-making in patient risk assessment using a hierarchical fuzzy model. Cognition, Technology and Work, 20, 477-488.

Kara, A., \& Dogan, I. (2018). Reinforcement learning appraoches for specifying ordering policies of perishable inventory systems. Expert Systems With Applications, 91, 150-158. 
Khalil, J. (2010). A Cost Optimisation Aproach for the Storage of Spare Parts Within an Industrial Environment. 20th International Conference on Flexible Automation and Intelligent Manufacturing (p. ). San Francisco: FAIM 2010.

Komal. (2019). Fuzzy reliability analysis of the compressor house unit system in a coal fired thermal power plant using TBGFLT technique. International Journal of Quality \& Reliability Management, 3(5), 686-707.

L, T., \& Saaty, T. L. (1990). How to make a decision: The analytic hierarchy process. European Journal of Operational Research, 48(1), 9-26.

Labib, A. W. (2011). A supplier selection model: A comparison of fuzzy logic on the Analytical Hierarchy Process. International Journal of Production Research, 49.

Lengu, D., Syntetos, A. A., \& Babai, M. Z. (2014). Spare parts management: Linking distributional assumptions to demand classification. European Journal of Operation Research, 235, 624-635.

Lopez-Soto, D., Angel-Bello, F., Yacout, S., \& Alvarez, A. (2017). A mutli-start algorithm to design a multi-class classifier for a multi-criteria ABC inventory classification problem. Experts System With Applications, 81, $12-21$.

Moharana, U. C., \& Sarmah, S. P. (2016). Joint replenishment of associated spare parts using clustering approach. Int J Adv Manuf Technol, 94, 2535-2549.

Moharana, U., Sarmah, S., \& Rathore, P. K. (2019). Application of data mining for spare parts information in maintenance schedule: a case study. International Journal of Quality \& Reliability Management, 10551072.

Mostafa, S., Dumrak, J., \& Soltan, H. (2015). Lean Maintenance Roadmap. Procedia Manufacturing. 2, pp. 434444. ScienceDirect.

Naji, A., El Oumami, M., Bouksour, O., \& Beidouri, Z. (2019). A mixed methods research toward a framework of a maintenance management model. Journal of Quality in Maintenance Engineering, 260-289.

Prak, D. R., Teunter, R., \& Riezebos, J. (2014). Periodic review and continuous ordering. Groningen: SOM Research Reports.

Prak, D., Ruud, T., \& Syntetos, A. (2017). On the calculation of safety stocks when demand is forecasted. European Journal of Operational Research, 256, 454-461.

Prak, D., Teunter, R. H., Babai, M. Z., Syntetos, A. A., \& Boylan, J. E. (2018). Forecasting and Inventory Control with Compund Poisson Demand Using Periodic Demand Data. Groningen: SOM Research Reports.

Qiwei, H., Boylan, J. E., Huijing, C., \& Labib, A. (2018). OR in spare aprts management: A review. European Journal of Operational Research, 266(2), 395-414.

Rashid, H. S., Place, C. S., \& Braithwaite, G. R. (2014). Eradicating root causes of aviation maintenance errors: introducing the AMMP. Cognition, Technology, Work, 16(1), 71-90. 
Rekik, Y., Christoph, H. G., \& Syntetos, A. A. (2017). Enriching demand forecasts with managerial information to improve inventory replineshment decisions: Exploiting judgment and fostering learning. European Journal of Operational Research, 261, 182-194.

Samima, S., Sarma, M., Samanta, D., \& Prasad, G. (2019). Estimation and quantification of vigilance using ERPs and eye blink rate with a fuzzy model-based approach. Cognition Technology \& Work, 21, 517-533.

Schuh, P., Schnider, D., Funke, L., \& Tracht, K. (2015). Cost-Optimal spare parts inventory planning for wind energy systems. Logistics Research.

Sene, A., Kamsu-Foguem, B., \& Rumeau, P. (2018). Decision support system for in-flight emergency events. Cognition, Technology \& Work, 20(2), 245-266.

Sleptchenko, A., \& Van der Heijden, M. (2016). Joint optimization of redundancy level and spare part inventories. Reliability Engineering and System Safety, 153.

Somarin, A. R., Chen, S., Asian, S., \& Wang, D. Z. (2017). A heuritic stock allocation rule for repairable service parts. International Journal of Production Economics, 131-140.

Stoll, J., Kopf, R., \& Lanza, G. (2015). Criticality analysis of spare parts management: a multi criteria classification regarding a cross-plant central warehouse strategy. Production Engineering, 9, 225-235.

Syntetos, A. A., \& Teunter, R. H. (2014). On the calcualtion of safety stocks. Groningen: SOM Research Reports.

Syntetos, A. A., Teunter, R. H., Babai, M. Z., \& Transchel, S. (2016). On the benefits of delayed ordering. European Journal of Operatioal Research, 248, 963-970.

Teixeira, C., Lopes, I., \& Figueiredo, M. (2017). Multi-criteria classification for spare parts management: a case study. Flexible Automation and Intelligent Manufacturing 2017 (pp. 1560-1567). Modena, Italy: Procedia Manufacturing.

Tillement, S., \& Hayes, J. (2019). Maintenance schedules as boundary objects for improved organizational reliability. Cognition, Technology \& Work, 21(3), 497-515.

Van der Auweraer, S., Boute, R. N., \& Syntetos, A. A. (2019). Forecasting spare part demand with installe dbase information: A review. International Journal of Forecasting, 35, 181-196.

Wen, M., Han, Q., Yang, Y., \& Kang, R. (2017). Uncertain optimization model for multi-echelon spare parts supply system. Applied Soft Computing.

Yoo, S. H., \& Kim, B. C. (2016). Joint pricing of new and refurbushed items: A comparison of closeed-loop supply chain model. Int. J. Production Economics, 132-143.

Zada, L. (1965). Fuzzy sets. Information and control , 8(3), 338-353.

Zahedi-Hooseini, F., Scarf, P., \& Syntetos, A. (2017). Joint Optimisation of inspection maintenance and spare parts provisioning: a comparative study of inventory policies using simulation and survey data. Reliability Engineering and System Safety, 168, 306-316. 
Zahedi-Hosseini, F., Scarf, P., \& Syntetos, A. (2018). Joint mainteannce-inventory optimisation of parallel production systems. Journal of Manufacturing Systems, 48, 73-86.

Zare, A., Malakoutikhah, M., \& Alimohammadou, M. (2019). Selecting lighting system based on workers' cognitive performance using fuzzy best-worst method and Qualiflex. Cognitive Technology \& Work, 1-12.

Zu, S., Dekker, R., Willem, V., Renjie, R. W., \& Koning, A. J. (2017). An Improved method for forecasting spare parts demand using extreme value theory. European Journal of Operational Research, 261(1), 169-181.

\section{Appendix I: Rule Base Tables}

Tables

\section{Appendix II}

Table A. II.I

\section{The numerical expression of the extreme ratios}

As per the tables in Appendix I, the domain of extreme ratios is between $(1: 2)$ and $(1: 10)$. The smallest variation in ratios is 0.05 , therefore the whole domain being equal to 8 may be divided into 160 step. On the other hand the 160 step should be fitted between fuzzy set values FSV 0.05 and 0 on one side and 9.5 and 10 on the other side. The result is a FSV increment variation of $0.0003125 /$ step. Given these inputs the second table is built.

Table A. II.II 